\title{
Electrostatic and Small-Signal Analysis of CMUTs With Circular and Square Anisotropic Plates
}

la Cour, Mette Funding; Christiansen, Thomas Lehrmann; Jensen, Jørgen Arendt; Thomsen, Erik Vilain

Published in:

IEEE Transactions on Ultrasonics, Ferroelectrics and Frequency Control

Link to article, DOI:

10.1109/TUFFC.2014.006553

Publication date:

2015

Document Version

Peer reviewed version

Link back to DTU Orbit

Citation (APA):

la Cour, M. F., Christiansen, T. L., Jensen, J. A., \& Thomsen, E. V. (2015). Electrostatic and Small-Signal Analysis of CMUTs With Circular and Square Anisotropic Plates. IEEE Transactions on Ultrasonics, Ferroelectrics and Frequency Control, 62(8), 1563-1579. https://doi.org/10.1109/TUFFC.2014.006553

\section{General rights}

Copyright and moral rights for the publications made accessible in the public portal are retained by the authors and/or other copyright owners and it is a condition of accessing publications that users recognise and abide by the legal requirements associated with these rights.

- Users may download and print one copy of any publication from the public portal for the purpose of private study or research.

- You may not further distribute the material or use it for any profit-making activity or commercial gain

- You may freely distribute the URL identifying the publication in the public portal 


\title{
Electrostatic Analysis of CMUTs with Circular and Square Anisotropic Plates
}

\author{
Mette Funding la Cour*†, Thomas Lehrmann Christiansen ${ }^{\dagger}$ Jørgen Arendt Jensen* and Erik Vilain Thomsen ${ }^{\dagger}$ \\ ${ }^{*}$ Center for Fast Ultrasound Imaging, Department of Electrical Engineering, Technical University of Denmark, \\ DK-2800 Kgs. Lyngby, Denmark \\ ${ }^{\dagger}$ Department of Micro and Nanotechnology, Technical University of Denmark, DK-2800 Kgs. Lyngby, Denmark
}

\begin{abstract}
Traditionally, Capacitive Micromachined Ultrasonic Transducers (CMUTs) are modeled using the isotropic plate equation and this leads to deviations between analytical calculations and Finite Element Modeling (FEM). In this paper, the deflection is calculated for both circular and square plates using the full anisotropic plate equation. It is shown that the anisotropic calculations match perfectly with FEM while an isotropic approach causes up to $10 \%$ deviations in deflection. For circular plates an exact solution can be found and for square plates using the Galerkin method and utilizing the symmetry of the silicon crystal, a compact and accurate expression for the deflection can be obtained. The deviation from FEM in center deflection is $<0.1 \%$. The theory of multilayer plates is also applied to the CMUT. The deflection of a square plate was measured on fabricated CMUTs using a white light interferometer. Fitting the plate parameter for the anisotropic calculated deflection to the measurement, a deviation of $0.07 \%$ is seen. Furthermore, electrostatic analysis is performed using energy considerations and the calculated deflections to include the anisotropy. The stable position, effective spring constant, pull-in distance and pull-in voltage are found for both circular and square anisotropic plates and the pressure dependence is also included by comparing to the corresponding analysis for a parallel plate. Finally, it was also measured how fabricated devices with both circular and square plates behaved under increasing bias voltage and it is observed that the models including anisotropic effects are within the uncertainty interval of the measurements.
\end{abstract}

\section{INTRODUCTION}

Precise modeling of capacitive micromachined ultrasonic transducers (CMUT) is important for an efficient design process. A CMUT consists of two plates where one of them is fixed and the other can deflect. The deflection $w(x, y)$ of the movable plate is an important parameter that influences several basic CMUT parameters such as pull-in voltage and capacitance. Most existing analytical approaches use the isotropic plate equation to calculate the deflection i.e. [1], [2]. However, when using fusion bonding fabrication technology [3], the plate usually consists of crystalline silicon. Having a silicon (001) substrate, which are most often used, Young's modulus and Poisson's ratio are strongly anisotropic. The isotropic approach is then not correct, and can result in deviations in the deflection compared to finite element modeling (FEM) taking the anisotropy into account and measurements. Therefore, to get precise modeling of these CMUTs, the anisotropy of silicon needs to be taken into account.

The first decade after CMUTs were invented, various analytical models were presented for circular cells, which included more and more features of the device behaviour [4], [5], [6]. However, all of them were based on parallel plate approximations for the deflection leading only to estimates of the critical CMUT parameters. Later, the actual deflection of the movable plate clamped at the edges was taken into account [2], [7], [8], where it was used for calculating pull-in voltage and derivation of an equivalent circuit model. The effect from having a nonuniform load on the plate was included in solving the plate equation by [7] who used superposition and a concentrically loaded plate, and by [9] who used the Galerkin method. The non-uniform load occurs when the bias voltage is increased, as the electrostatic force will be greater where the gap is smaller. This effect gets more distinct when the deflection is larger. However, it is not necessary to include for the typical CMUT case, where the plate never deflect more than half the gap due to pull-in.

All of these models assumes a circular plate geometry of the CMUT cells. For circular plates, a simple and exact solution for the deflection exists [10], but this is not the case for square plates. Existing solutions for the deflection of square plates is based on series expansions with either trigonometric [11] or polynomial basis functions [12]. None of these, however, take the anisotropy of the plate into account.

For the first fabricated CMUTs there was no need for using anisotropic plate theory, as the plate usually consisted of silicon nitride or polysilicon when fabricated with the sacrificial release method. After the fusion bonding fabrication method was applied to CMUTs, the anisotropy of the plate, which now consisted of crystalline silicon, was considered [13]. However, instead of solving the problem analytically, FEM was used to estimate a set of material parameters (Young's modulus and Poisson's ratio) to use in the models to get an approximation as close as possible to the correct anisotropic solution.

This paper presents solutions to the full anisotropic plate equation for both circular and square plates used in fusion bonded CMUTs. The models were initially presented in [14] for an anisotropic plate with circular geometry, and this was then modified and expanded to include square plates as well in [15]. For the circular cells the symmetry reduces the plate equation and an exact solution for the anisotropic case can be obtained similarly to the isotropic solution. The approach used to solve the equation for the square plate is the Galerkin method [16]. Utilizing the symmetry of the silicon crystal, a compact and precise approximation of the deflection of a square plate can be obtained for the anisotropic case. 
The plate usually also consists of more than one material. The theory of laminar plates is described in [17], [18] and in this paper, the multilayer plate theory including anisotropy is applied to calculate important parameters for a two layer silicon/metal plate typically used for CMUTs.

Having found the deflection of the CMUT plate, a model for the electrostatic behavior of the transducer can be set up. Circular cells have been investigated thoroughly during the years and a full model for this plate geometry has recently been presented [8], whereas the full electrostatic analysis for the square plate has not been investigated previously. Furthermore, none of the existing models include the anisotropy.

The isotropic plate equation and solutions for circular and square plates can be found in Section II. It is followed by the anisotropic plate equation and how to utilize the symmetry of the silicon crystal to reduce and solve the problem in Section III for both circular and square plates. The calculated deflection is compared to the solution for corresponding isotropic cases, FEM, and measurements performed on fabricated devices. Furthermore, in Section VI the calculated deflection is used to find the stable position, effective spring constant, pull-in distance and pull-in voltage of the CMUT plates. The pressure dependence is also included. Measurements of the stable position are performed on devices with both circular and square plates and the theory is compared to these in Section VII.

\section{THE ISOTROPIC PLATE EQUATION}

Conventionally, the deflection $w(x, y)$ of a CMUT with a thin plate is modeled using the isotropic plate equation [11]

$$
\frac{\partial^{4} w}{\partial x^{4}}+2 \frac{\partial^{4} w}{\partial x^{2} \partial y^{2}}+\frac{\partial^{4} w}{\partial y^{4}}=\frac{p}{D_{\mathrm{i}}},
$$

where $p$ is the applied pressure difference across the plate and the flexural rigidity is given by

$$
D_{\mathrm{i}}=\frac{E}{12\left(1-\nu^{2}\right)} h^{3},
$$

with $E$ being Young's modulus, $\nu$ being Poisson's ratio, and $h$ being the thickness of the plate.

For thin clamped circular plates, an exact solution exists. For such a plate with radius $a$, the center deflection is given by [10]

$$
w_{0, \text { iso }, \text { circ }}=\frac{1}{64} \frac{a^{4} p}{D_{\mathrm{i}}} .
$$

For clamped rectangular and square plates, no simple exact solution exists and approximate methods have to be used. The conventional isotropic approach is based on a series expansion of the deflection, and the center deflection for a thin clamped square plate having side length $2 L$ is [11]

$$
w_{0, \text { iso }, \text { sq }}=0.020245 \frac{L^{4} p}{D_{\mathrm{i}}} .
$$

Fig. 1 shows a cross sectional view of a CMUT cell with an applied voltage. The device parameters are illustrated for both circular and square plates.

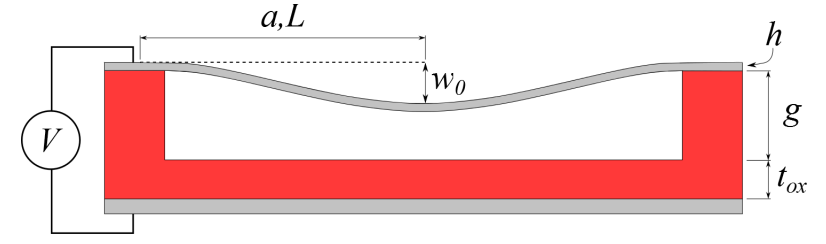

Fig. 1. Cross sectional view of CMUT cell with applied voltage.

TABLE I

ROOM TEMPERATURE (300K) COMPLIANCE COEFFICIENTS FOR N-TYPE CRYSTALLINE SILICON MEASURED BY [21] FOR A SUBSTRATE WITH LOW DOPING LEVEL $\left(150 \Omega\right.$-CM, $\left.\sim 2.8 \times 10^{13} \mathrm{CM}^{-3}\right)$ AND HIGH DOPING LEVEL $\left(3.26 \mathrm{M} \Omega\right.$-CM, $\left.\sim 2.1 \times 10^{19} \mathrm{CM}^{-3}\right)$.

\begin{tabular}{ccc}
\hline \hline & Low doping & High doping \\
\hline$s_{11}^{\mathrm{c}}$ & $7.691 \times 10^{-12} \mathrm{~Pa}^{-1}$ & $7.858 \times 10^{-12} \mathrm{~Pa}^{-1}$ \\
$s_{12}^{\mathrm{c}}$ & $-2.1420 \times 10^{-12} \mathrm{~Pa}^{-1}$ & $-2.2254 \times 10^{-12} \mathrm{~Pa}^{-1}$ \\
$s_{44}^{\mathrm{c}}$ & $12.577 \times 10^{-12} \mathrm{~Pa}^{-1}$ & $12.628 \times 10^{-12} \mathrm{~Pa}^{-1}$ \\
\hline \hline
\end{tabular}

\section{THE ANISOTROPIC PLATE EQUATION}

To take the anisotropy of the plate into account and avoid the inaccuracy from isotropic modeling, the stiffness of the plate needs to be described through the stiffness matrix of the material instead of Young's modulus and Poisson's ratio. The starting point is the relation between stress, $\sigma$, and strain, $\epsilon$, [19]

$$
\sigma^{\mathrm{c}}=\mathbf{c}^{\mathrm{c}} \epsilon^{\mathrm{c}}, \text { or } \epsilon^{\mathrm{c}}=\mathbf{s}^{\mathrm{c}} \sigma^{\mathrm{c}} .
$$

Here superscript $\mathrm{c}$ denotes the crystallographic coordinate system, so $\mathbf{c}^{\mathrm{c}}$ is the stiffness matrix and $\mathbf{s}^{\mathrm{c}}=\left(\mathbf{c}^{\mathrm{c}}\right)^{-1}$ the compliance matrix in this coordinate system. Having a thin plate, the stresses in the $z$ direction can be ignored and plane stress assumed. Using the voigt notation, the relation between strain and stress then becomes [20]

$$
\left(\begin{array}{c}
\varepsilon_{1} \\
\varepsilon_{2} \\
\varepsilon_{6}
\end{array}\right)=\left(\begin{array}{lll}
s_{11} & s_{12} & s_{16} \\
s_{12} & s_{22} & s_{26} \\
s_{16} & s_{26} & s_{66}
\end{array}\right)\left(\begin{array}{c}
\sigma_{1} \\
\sigma_{2} \\
\sigma_{6}
\end{array}\right)=\mathbf{S}_{\mathrm{eff}}\left(\begin{array}{c}
\sigma_{1} \\
\sigma_{2} \\
\sigma_{6}
\end{array}\right),
$$

and we can define an effective stiffness matrix from the effective compliance matrix

$$
\mathbf{C}_{\text {eff }}=\left(\mathbf{S}_{\text {eff }}\right)^{-1} \text {. }
$$

For silicon the effective compliance matrix is

$$
\mathbf{S}_{\text {eff }}^{\mathrm{c}}=\left(\begin{array}{ccc}
s_{11}^{\mathrm{c}} & s_{12}^{\mathrm{c}} & 0 \\
s_{12}^{\mathrm{c}} & s_{11}^{\mathrm{c}} & 0 \\
0 & 0 & s_{44}^{\mathrm{c}}
\end{array}\right) .
$$

The elements in this matrix are known from measurements and shown in Table I [21]. It is noted that the elements in (8) are known in the crystallographic coordinate system, which is not necessarily the same as the coordinate system of the plate. To illustrate this further, the crystallographic and the plate coordinate systems can be seen in Fig. 2. The solid coordinate 
system aligned to the $\langle 100\rangle$ directions is where the compliance values for silicon are known and the dashed system shows the rotated coordinate system for the plate where the compliance values needs to be calculated. Having silicon as plate material and performing standard cleanroom fabrication, the plate will usually be on a (001) substrate and aligned to the primary wafer flat. Flat alignment is to the [110] direction, so the plate coordinate system will be rotated $\psi=45^{\circ}$ with respect to the crystallographic coordinate system. A transformation of the compliance matrix between the two coordinate systems is therefore needed. As it is the stiffness matrix elements that are to be used in the plate equation, the resulting effective stiffness matrix for the present case can be expressed through (7) $[20]$

$$
\begin{aligned}
& C_{\mathrm{Si}(001),[110]}^{\mathrm{eff}}= \\
& \left(\begin{array}{ccc}
\frac{1}{s_{44}^{\mathrm{c}}}+\frac{1}{2\left(s_{11}^{\mathrm{c}}+s_{12}^{\mathrm{c}}\right)} & \frac{1}{2\left(s_{11}^{\mathrm{c}}+s_{12}^{\mathrm{c}}\right)}-\frac{1}{s_{44}^{\mathrm{c}}} & 0 \\
\frac{1}{2\left(s_{11}^{\mathrm{c}}+s_{12}^{\mathrm{c}}\right)}-\frac{1}{s_{44}^{\mathrm{c}}} & \frac{1}{s_{44}^{\mathrm{c}}}+\frac{1}{2\left(s_{11}^{\mathrm{c}}+s_{12}^{\mathrm{c}}\right)} & 0 \\
0 & 0 & \frac{1}{2 s_{11}^{\mathrm{c}}-2 s_{12}^{\mathrm{c}}}
\end{array}\right) .
\end{aligned}
$$

It is seen that the stiffness matrix has an orthotropic symmetry.

Having the effective stiffness matrix, the generalized plate equation can be used. This is a differential equation for the deflection, $w(x, y)$, of a thin anisotropic plate exposed to a uniform load $p$ given by [20], [22]

$\frac{\partial^{4} w}{\partial x^{4}}+k_{1} \frac{\partial^{4} w}{\partial x^{3} \partial y}+k_{2} \frac{\partial^{4} w}{\partial x^{2} \partial y^{2}}+k_{3} \frac{\partial^{4} w}{\partial x \partial y^{3}}+k_{4} \frac{\partial^{4} w}{\partial y^{4}}=\frac{p}{D_{a}}$.

The plate coefficients $k_{1}-k_{4}$ and the anisotropic flexural rigidity, $D_{\text {a }}$, depend on the elastic constants of the plate material

$$
\begin{gathered}
k_{1}=\frac{4 C_{13}^{\text {eff }}}{C_{11}^{\text {eff }}} \quad k_{2}=\frac{2\left(C_{12}^{\text {eff }}+2 C_{33}^{\text {eff }}\right)}{C_{11}^{\text {eff }}} \quad k_{3}=\frac{4 C_{23}^{\text {eff }}}{C_{11}^{\text {eff }}} \\
k_{4}=\frac{C_{22}^{\text {eff }}}{C_{11}^{\text {eff }}} \quad D_{\mathrm{a}}=\frac{1}{12} h^{3} C_{11}^{\text {eff }},
\end{gathered}
$$

where $C_{p q}^{\text {eff }}$ are elements in the effective stiffness matrix (9). Note that the stiffness of the plate is no longer expressed through Young's modulus and Poisson's ratio but directly through the stiffness values.

Using the compliance values for silicon (Table I) and inserting the stiffness elements in (9) into (11), it follows that $k_{1}=k_{3}=0$ and $k_{4}=1$. Thus, aligning the plate to the primary flat simplifies the anisotropic plate equation (10) to

$$
\frac{\partial^{4} w}{\partial x^{4}}+k_{2} \frac{\partial^{4} w}{\partial x^{2} \partial y^{2}}+\frac{\partial^{4} w}{\partial y^{4}}=\frac{p}{D_{\mathrm{a}}} .
$$

The same is the case for aligning the plate along the [100] direction where the inverse of (8) is used instead of (9), resulting in the same values for $k_{1}, k_{3}$ and $k_{4}$. For these two special cases, the coefficients in the plate equation are summarized in Table II for both high and low doping levels of the substrate.

\section{A. Deflection of Circular plates}

The solution to (10) for a circular plate of radius $a$ fixed at the boundary is easily obtained using polar coordinates. The

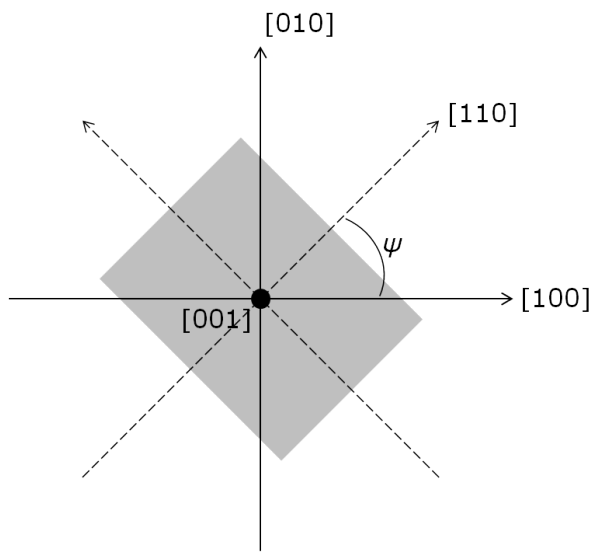

Fig. 2. The two coordinate systems, solid lines are the crystallographic system aligned to the $\langle 100\rangle$ direction and the dashed lines the plate system aligned to the $\langle 110\rangle$ direction.

TABLE III

YOUNG'S MODULUS AND POISSON'S RATIO FOR SILICON (001).

\begin{tabular}{ccc}
\hline \hline & Young's modulus & Poisson's ratio \\
\hline [100] direction & $130 \mathrm{GPa}$ & 0.278 \\
[110] direction & $169 \mathrm{GPa}$ & 0.062 \\
Mean value & $148 \mathrm{GPa}$ & 0.177 \\
\hline \hline
\end{tabular}

deflection at a point a distance $r$ from the center is given by [10]

$$
\frac{w(r)}{w_{0}}=\left(1-\left(\frac{r}{a}\right)^{2}\right)^{2} .
$$

This expression is similar to the deflection for the isotropic case, however, the center deflection is different

$$
w_{0, \text { circ }}=\frac{1}{8\left(3+k_{2}+3 k_{4}\right)} \frac{a^{4} p}{D_{\mathrm{a}}} .
$$

By combining (3) and (14) it is possible to find an effective flexural rigidity

$$
D_{\mathrm{eff}}=\frac{3+k_{2}+3 k_{4}}{8} D_{\mathrm{a}} .
$$

This can be used to easily change from the isotropic plate equation to the anisotropic plate equation in already existing analytical models of CMUTs. An example of this will be shown in section VI. Using the plate coefficient values from Table II for a highly doped (001) silicon plate aligned to the $\langle 110\rangle$ direction, the effective flexural rigidity becomes $D_{\text {eff }}=0.91551 D_{\text {a }}$.

To compare the anisotropic model with the isotropic approach and FEM simulations, the normalized deflection of a CMUT exposed to a pressure difference is shown in Fig. 3. The FEM simulations were performed in COMSOL Multiphysics version 4.2a using the full anisotropic stiffness tensor and the curves are normalized to the center deflection of this. The isotropic curves are made using (13) and (3) and Young's modulus and Poisson's ratio along the [100] and [110] directions (see Table III) to give the dash and dashdot lines, respectively. The anisotropic solution is made using (13) 
TABLE II

SELECTED VALUES FOR THE PLATE COEFFICIENTS AND ANISOTROPIC FLEXURAL RIGIDITY FOR PLATES ON A SILICON (001) SUBSTRATE [20]. UPPER VALUES IN BRACKETS ARE FOR LOW DOPING LEVEL AND LOWER VALUES FOR HIGH DOPING LEVEL.

\begin{tabular}{|c|c|c|c|c|c|c|}
\hline Orientation & $\psi$ & $k_{1}$ & $k_{2}$ & $k_{3}$ & $k_{4}$ & $12 D_{a} / h^{3}[\mathrm{GPa}]$ \\
\hline \multirow{2}{*}{ [100] } & \multirow{2}{*}{0} & \multirow{2}{*}{0} & $2.8133 \pm 0.0006$ & \multirow{2}{*}{0} & \multirow{2}{*}{1} & $140.96 \pm 0.03$ \\
\hline & & & $2.8559 \pm 0.0006$ & & & $138.35 \pm 0.03$ \\
\hline \multirow{2}{*}{ [110] } & \multirow{2}{*}{$\pi / 4$} & \multirow{2}{*}{0} & $1.3241 \pm 0.0004$ & \multirow{2}{*}{0} & \multirow{2}{*}{1} & $169.62 \pm 0.03$ \\
\hline & & & $1.2949 \pm 0.0004$ & & & $167.96 \pm 0.03$ \\
\hline
\end{tabular}

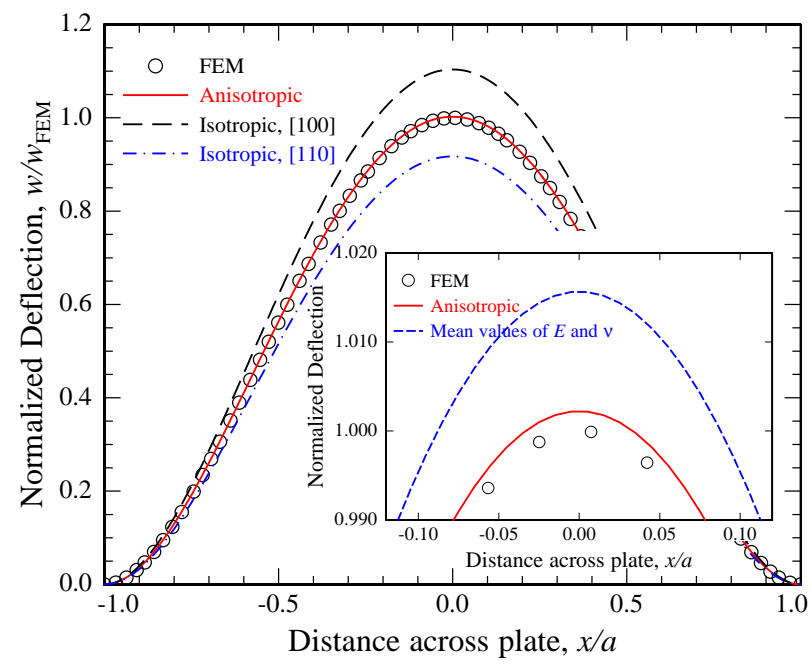

Fig. 3. Normalized deflection cross section $(y=0)$ of a thin circular plate of silicon (001) calculated with (13) using both the isotropic approach (3) with Young's modulus and Poisson's ratio in the [100] and [110] directions and the anisotropic approach (14). The circles represent the deflection calculated by FEM.

and (14) and is shown as a solid curve. This is on top of the FEM simulation (circles). Due to the symmetry of the circular plate, any set of parameters from Table II can be used. Excellent agreement between the anisotropic solution and the finite element calculation is seen with an error of less than $0.3 \%$, which is due to grid size and slightly different boundary conditions. The figure also shows that using Young's modulus and Poisson's ratio corresponding to [100] or [110] directions lead to errors in the center deflection of around $10 \%$. To reduce this error, it is common practice to use mean values of Young's modulus and Poisson's ratio (see Table III) which decreases the error to around $1.5 \%$. As it can be hard to see the solutions with small deviations from the FEM, a zoom in on the center region of the plate is shown in the insert in Fig. 3. Here the reduction in deviation from FEM by using the anisotropic solution compared to the isotropic mean values of Young's modulus and Poisson's ratio is clearly visible. Using the anisotropic approach for a thin circular CMUT plate on a (001) silicon substrate is simple and the result is exact.

\section{B. Deflection of Square Plates}

Having a square plate makes analytical deflection calculations complicated and approximate methods must be used to solve the anisotropic plate equation. With the anisotropic approach, the Galerkin method [16] can be used to find approximate expressions for the deflection of a thin anisotropic square plate. As previously stated, in the most common case for CMUTs, the plate is fabricated on a silicon (001) substrate and aligned to the [110] direction. For this orthotropic square plate with sidelengths $2 L$, the relative deflection is found to be [20], [23]

$$
\begin{aligned}
\frac{w(x, y)}{w_{0}} & =\left[1-\left(\frac{x}{L}\right)^{2}\right]^{2}\left[1-\left(\frac{y}{L}\right)^{2}\right]^{2} \\
& \times\left[1+\beta\left(\frac{x}{L}\right)^{2}+\beta\left(\frac{y}{L}\right)^{2}\right],
\end{aligned}
$$

where the plate parameter $\beta$ is defined as

$$
\beta=\frac{182+143 k_{2}}{1432+91 k_{2}} \text {. }
$$

The center deflection can be written

$$
w_{0, \mathrm{sq}, \mathrm{Si}(001)}=\frac{77\left(1432+91 k_{2}\right)}{256\left(16220+11 k_{2}\left(329+13 k_{2}\right)\right)} \frac{L^{4} p}{D_{\mathrm{a}}} .
$$

Equations (16)-(18) are also valid when the plate is aligned to the [100] direction on a silicon (001) substrate. Note that the center deflection depends only on the $k_{2}$ coefficient. For primary flat alignment, it is found by inserting $k_{2}$ into (17) and using the low doping values $\beta_{\text {low }}=0.23920$ and using the high doping values $\beta_{\text {high }}=0.23691$. For the low doping case, this results in a normalized deflection for the plate aligned to the $\langle 110\rangle$ direction given by

$$
\begin{aligned}
\left.\frac{w(x, y)}{w_{0}}\right|_{\mathrm{sq}, \operatorname{Si}(001),\langle 110\rangle} & =\left[1-(x / L)^{2}\right]^{2}\left[1-(y / L)^{2}\right]^{2} \\
& \times\left[1+0.23920\left[(x / L)^{2}+(y / L)^{2}\right]\right],
\end{aligned}
$$

and the center deflection becomes

$$
w_{0, \mathrm{sq}, \mathrm{Si}(001),\langle 110\rangle}=0.02196 \frac{L^{4} p}{D_{\mathrm{a}}} .
$$

For the high doping case, the factor in front becomes 0.02204 for the center deflection. Comparing (4) and (20), it is seen that they are very similar containing the same parameters but different coefficients and the anisotropic instead of the isotropic flexural rigidity.

Fig. 4 shows the deflection cross section through $y=0$ of a square plate of silicon (001) given by the reduced version of (16)

$$
w_{y=0, \mathrm{sq}}=w_{0}\left[1-(x / L)^{2}\right]^{2}\left[1+\beta(x / L)^{2}\right] .
$$




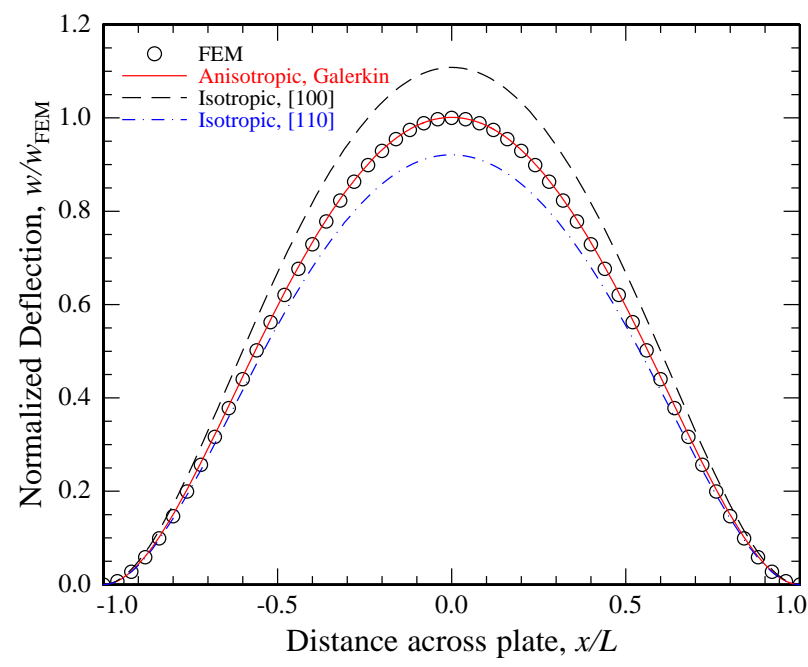

Fig. 4. Normalized deflection cross section $(y=0)$ of a square plate of silicon (001) calculated with (21) using both the isotropic approach (4) with Young's modulus and Poisson's ratio in the [100] and [110] directions and the anisotropic approach (20). The circles represent the deflection calculated by FEM.

The deflection calculated with the anisotropic approach uses $k_{2}=1.3241$ in (17) and center deflection (20) (solid curve). This is compared to the isotropic approach using $k_{2}=2$ in (17) and center deflection (4), with Young's modulus and Poisson's ratio in the [100] and [110] directions (dash and dashdot curves), and to a finite element (FEM) simulation made using the full anisotropic compliance matrix (compliance coefficients from Table I) in COMSOL (circles). The calculated deflections are normalized to the FEM center deflection. Excellent agreement is shown between the anisotropic curve and FEM with a deviation of less than $0.1 \%$ whereas the isotropic approach leads to deviations in the center deflection of around $10 \%$ for both [100] and [110] directions.

\section{Multilayer Plates}

Following the method by [17], [18] the anisotropic plate theory can be expanded to also include plates consisting of more than one layer. Starting from equations for the moment and stress resultants, it can be found that the general plate equation including anisotropic effects has the same form as for the single layer plate (10), however, the plate coefficients $k_{1}-k_{4}$ and the plate stiffness $D_{\mathrm{a}}$ will be different to capture effects from having a multilayer plate.

For the CMUT application, the multilayer plate will often consist of two layers with silicon as the main part and a thin aluminum layer on top for contacts. The aluminum is an isotropic material and the silicon is, as seen on (8), an orthotropic material (when aligned to [110] direction on a (001) substrate). For this two-layer plate, the total thickness is called $h$ and the ratio $\alpha=h_{\mathrm{Al}} / h$ is defined from the thickness of the aluminum, $h_{\mathrm{Al}}$. When the plate is all silicon $\alpha=0$ and when the plate is only aluminum $\alpha=1$. Again utilizing the symmetry of the materials, it can be found that $k_{1}=k_{3}=0$ and $k_{4}=1$ so again only $k_{2}$ and $D_{\mathrm{a}}$ need to be taken into account for the usual CMUT plates.

The expressions for $k_{2}$ and the plate stiffness becomes quite long even for the simplified case. Using the compliance values for highly doped silicon in Table I and Young's modulus of $E=70 \mathrm{GPa}$ and Poisson's ratio of $\nu=0.35$ for aluminum in the expressions, they become

$$
\begin{aligned}
D_{\mathrm{AlSi}} & =(13.9963 \mathrm{GPa}-22.0458 \mathrm{GPa} \cdot \alpha) h^{3} \\
k_{2, \mathrm{AlSi}} & =1.29493+1.00464 \alpha .
\end{aligned}
$$

Furthermore, it can also be found that when having a sufficiently thin aluminum layer, $\alpha<0.2$, a series expansion can be used and simple correction formulas can be found. This way, the flexural rigidity of the combined aluminum and silicon plate compared to the flexural rigidity for a plate of only silicon with the same thickness as the total thickness can be expressed as

$$
\frac{D_{\mathrm{AlSi}}}{D_{\mathrm{Si}}}=1-1.575 \alpha \text {. }
$$

Similarly, for the plate parameter $k_{2}$ it is found that

$$
\frac{k_{2, \mathrm{AlSi}}}{k_{2, \mathrm{Si}}}=1-0.775822 \alpha \text {. }
$$

Equations (24) and (25) both use the stiffness values for highly doped silicon from Table I.

For a circular plate, the relative center deflection using the same method as above can be found to be

$$
\frac{w_{0, \mathrm{AlSi}, \mathrm{circ}}}{w_{0, \mathrm{Si}, \mathrm{circ}}}=1+1.437 \alpha \text {. }
$$

Doing the same for square plates the relation becomes

$$
\frac{w_{0, \mathrm{AlSi}, \mathrm{Sq}}}{w_{0, \mathrm{Si}, \mathrm{Sq}}}=1+1.445 \alpha \text {. }
$$

The error between the series expansion and the full result for the center deflection is less than $2 \%$ for $\alpha=0.2$ for both plate geometries. An example of a typical thicknesses of the layers of the CMUT multilayer plate is $\sim 2 \mu \mathrm{m}$ silicon and $\sim 0.2 \mu \mathrm{m}$ aluminum. This gives $\alpha=0.1$ and the error when using the series expansion is less than $0.5 \%$.

As examples on how the aluminum layer influences the plate parameter, stiffness and center deflection of the circular and square plates, calculations using single and multilayer plate theory can be seen in Table IV. Here, calculations are made with dimensions as the fabricated devices found in Table V. It is seen that including the aluminum layer in the calculations affects $k_{2}$ with around $7 \%$, the stiffness of the plate with around $18 \%$ and the center deflection with around $12 \%$ in this case.

\section{DEFLECTION MEASUREMENT}

To further validate the deflection of the square plate, CMUTs with square silicon plates have been fabricated using fusion bonding [24]. The dimensions of the fabricated device can be seen in Table V. The deflection was measured with a Sensofar PLu Neox 3D Optical Profiler using white light interferometry. Fig. 5 shows a measured cross section of the normalized deflection for a fabricated device. It is normalized 
TABLE IV

EXAMPLES ON $k_{2}$, STIFFNESS AND CENTER DEFLECTION WHEN USING SINGLE OR MULTILAYER PLATE THEORY.

\begin{tabular}{lcccc}
\hline \hline & $\alpha$ & $k_{2}$ & $12 D_{\mathrm{a}} / h^{3}$ & $w_{0}$ \\
\hline Circ., multi & 0.10 & 1.3954 & $141.50 \mathrm{GPa}$ & $29.4 \mathrm{~nm}$ \\
Circ., Si & - & 1.2949 & $167.96 \mathrm{GPa}$ & $25.7 \mathrm{~nm}$ \\
Sq., multi & 0.08 & 1.3753 & $146.79 \mathrm{GPa}$ & $12.5 \mathrm{~nm}$ \\
Sq., Si & - & 1.2949 & $167.96 \mathrm{GPa}$ & $11.2 \mathrm{~nm}$ \\
\hline
\end{tabular}

TABLE V

DIMENSIONS OF DEVICES FABRICATED WITH CIRCULAR AND SQUARE PLATES USING WAFER BONDING.

\begin{tabular}{lcc}
\hline \hline & Circular & Square \\
\hline Size $(a, L)$ & $36 \mu \mathrm{m}$ & $32.5 \mu \mathrm{m}$ \\
Plate thickness, Si $h_{\mathrm{Si}}$ & $1.8 \mu \mathrm{m}$ & $2.3 \mu \mathrm{m}$ \\
Al thickness $h_{\mathrm{Al}}$ & $200 \mathrm{~nm}$ & $200 \mathrm{~nm}$ \\
Gap height (vacuum) $g$ & (uncertain) & $405 \mathrm{~nm}$ \\
Insulation layer $t_{\mathrm{ox}}$ & $195 \mathrm{~nm}$ & $198 \mathrm{~nm}$ \\
\hline \hline
\end{tabular}

in both center deflection and distance across the plate to compare the shape of the measured deflection with the calculated deflection. The red curve is a fit made to the measurements using the anisotropic model (21). The plate parameter $\beta$ is fitted to the measurements. As it is seen in the figure, the fitted value for $\beta$ is 0.243 . Using (23) for calculating $\beta$ for this multilayer plate ( $2 \mu \mathrm{m}$ highly doped silicon (001) substrate aligned to [110] direction with $200 \mathrm{~nm} \mathrm{Al}$ ) a deviation of only $0.07 \%$ is obtained.

\section{Electrostatic Analysis}

Many important design parameters for CMUTs depend on the deflection of the plate. By using the solutions found in the

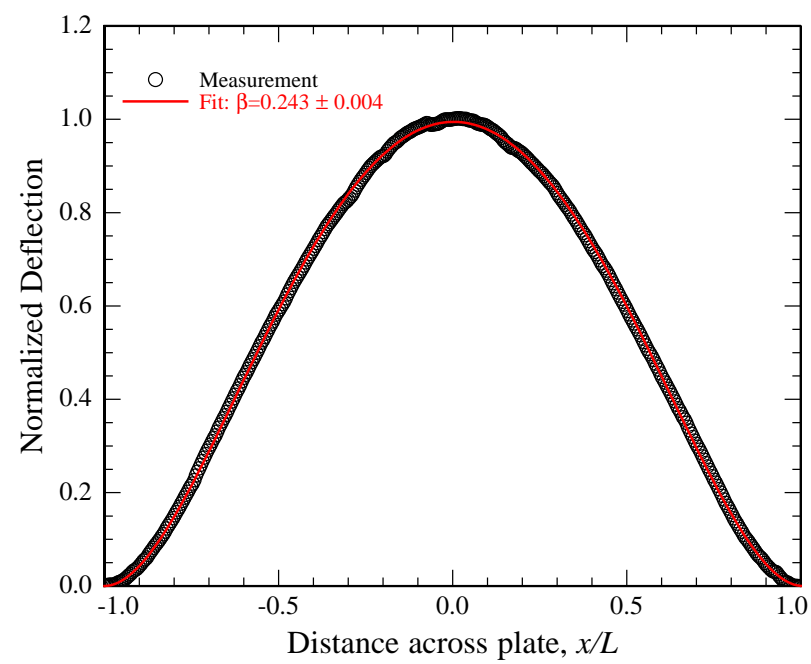

Fig. 5. Normalized deflection cross section $(y=0)$ from measurement on a fabricated CMUT with square plate of silicon (001) aligned to [110]. The red curve is a fit made from (21). previous sections and performing electrostatic analysis, it is possible to find the stable position of the plate, when applying a certain bias voltage. The stable position is the position where the spring force balances the electrostatic and pressure forces. From this the pull-in distance and pull-in voltage can be found.

The analysis in the following is based on energy considerations. The total potential energy $U_{\mathrm{t}}$ consists of three terms, the strain energy $U_{\mathrm{s}}$, the electrostatic energy $U_{\mathrm{e}}$, and the energy from applying a pressure $U_{\mathrm{p}}$

$$
U_{\mathrm{t}}=U_{\mathrm{s}}+U_{\mathrm{p}}+U_{\mathrm{e}}
$$

The method is valid for all systems where the total potential energy is of the form

$$
U_{\mathrm{t}}=\frac{k_{0} w_{0}^{2}}{2}-p A_{\mathrm{eff}} w_{0}-\frac{1}{2} V^{2} C_{\mathrm{t}}\left(w_{0}\right),
$$

where $k_{0}$ is the generalized spring constant that comes from the calculation of the strain energy, $A_{\text {eff }}$ is the effective area of the plates i.e. the area that goes into calculation of the work performed by deflecting the plate due to applied pressure, $V$ is the applied voltage, $p$ the atmospheric pressure, $C_{\mathrm{t}}$ the total capacitance of the device and $w_{0}$ the center deflection of the plate. For the parallel plate $k_{0}=k$ and $A_{\text {eff }}=A$.

The total force on the system, $F_{\mathrm{t}}$, is found by differentiating the total potential energy with respect to the center deflection, which is used as a reference in this work (any deflection could be used as a reference)

$$
F_{\mathrm{t}}=\frac{\partial U_{\mathrm{t}}}{\partial w_{0}}=k_{0} w_{0}-p A_{\mathrm{eff}}-\frac{1}{2} V^{2} C_{\mathrm{t}}^{\prime}\left(w_{0}\right),
$$

where $C_{\mathrm{t}}^{\prime}\left(w_{0}\right)$ denotes the capacitance differentiated with respect to $w_{0}$. The stable position of the plate can be found for a given applied voltage as the point where the total force is zero, so solving

$$
k_{0} w_{0}=p A_{\text {eff }}+\frac{1}{2} V^{2} C_{\mathrm{t}}^{\prime}\left(w_{0}\right) .
$$

The effective spring constant, $k_{\text {eff }}$, can be found as the second derivative of the total potential energy or by differentiating the total force

$$
k_{\text {eff }}=\frac{\partial F_{\mathrm{t}}}{\partial w_{0}}=k_{0}-\frac{1}{2} V^{2} C_{\mathrm{t}}^{\prime \prime}\left(w_{0}\right) .
$$

Pull-in occurs when the effective spring constant is zero and the pull-in voltage $V_{\mathrm{PI}}$ can be expressed as

$$
V_{\mathrm{PI}}=\sqrt{\frac{2 k_{0}}{C_{\mathrm{t}}^{\prime \prime}\left(w_{0}\right)}} .
$$

Inserting the pull-in voltage (33) into the equation for the stable position (31) the pull-in distance can be found by solving the equation

$$
k_{0} w_{0}=p A_{\mathrm{eff}}+\frac{k_{0} C_{\mathrm{t}}^{\prime}\left(w_{0}\right)}{C_{\mathrm{t}}^{\prime \prime}\left(w_{0}\right)} .
$$

This can then be inserted into (33) to obtain the pull-in voltage. Finding pull-in distance and voltage is therefore a question of solving the two equations (31) and (34) for the two variables.

In the following, this analysis is shown for both circular and square plates, with the anisotropic effects taken into account, 
and for a parallel plate capacitor for comparison. Similar analysis has previously been shown by others for isotropic circular plates i.e. [2], [8] and is therefore shown here in compact form with focus on the anisotropy of the plate.

\section{A. Capacitance}

An important variable in the electrostatic analysis for CMUTs is the capacitance. The capacitance at zero deflection, $C_{0}$, of the plate can for both the circular and square plates be divided into two contributions: The capacitance from the vacuum gap $C_{0}=\epsilon_{0} A / g$ and the capacitance from the insulation oxide between in electrodes $C_{\mathrm{ox}}=\epsilon_{0} \epsilon_{\mathrm{ox}} A / t_{\mathrm{ox}} \cdot A$ is the area of the plates, $g$ the vacuum gap, $\epsilon_{0}$ the vacuum permittivity, $t_{\mathrm{ox}}$ the thickness of the insulation oxide layer, and $\epsilon_{\mathrm{ox}}$ the relative permittivity of the oxide. The effect from having both contributions can be collected in an effective gap height

$$
g_{\mathrm{eff}}=g+\frac{t_{\mathrm{ox}}}{\epsilon_{\mathrm{ox}}} .
$$

The total capacitance at zero deflection can then be written

$$
C_{\mathrm{t} 0}=\left(\frac{1}{C_{0}}+\frac{1}{C_{\mathrm{ox}}}\right)^{-1}=\frac{\epsilon_{0} A}{g_{\mathrm{eff}}} .
$$

Taking the deflection of the plate into account, the total capacitance of the device is

$$
C_{\mathrm{t}}=\frac{1}{g_{\text {eff }}} \iint \frac{\epsilon_{0}}{1-\eta f(x, y)} \mathrm{d} x \mathrm{~d} y
$$

where $\eta=w_{0} / g_{\text {eff }}$ is the normalized center deflection and $f(x, y)$ is a function describing the shape of the deflection. For circular plates, this function will be (13), for square plates it is (19), and for the parallel plate $f=1$.

The total capacitance of a parallel plate capacitor is given by

$$
C_{\mathrm{t}, \text { parallel }}=C_{\mathrm{t} 0} \frac{1}{1-\eta} .
$$

For the circular plate, the integral can be solved analytically and the total capacitance becomes [2], [8]

$$
C_{\mathrm{t}, \mathrm{circ}}=C_{\mathrm{t} 0} \sqrt{\frac{1}{\eta}} \operatorname{arctanh} \sqrt{\eta} .
$$

For the square plate, there is no analytical solution. The integration in (37) can instead be performed numerically. The total capacitance for this plate can be written

$$
C_{\mathrm{t}, \mathrm{sq}}=C_{\mathrm{t} 0} f_{s}(\eta) \text {. }
$$

where $f_{s}(\eta)$ is a function describing the shape of the capacitance curve. Various functions can be used as the shape function, e.g. spline fit to the numerically found solution, a higher order polynomial fit or an interpolation function. A Taylor expansion can also be used, however, at least 8 terms is needed to get sufficient accuracy.

To obtain expressions that can be used for further calculations for the square plate, it is advantageous to look at where the capacitance is used and compare with results for the parallel plate. Inspecting (31)-(34) it is seen, that to find

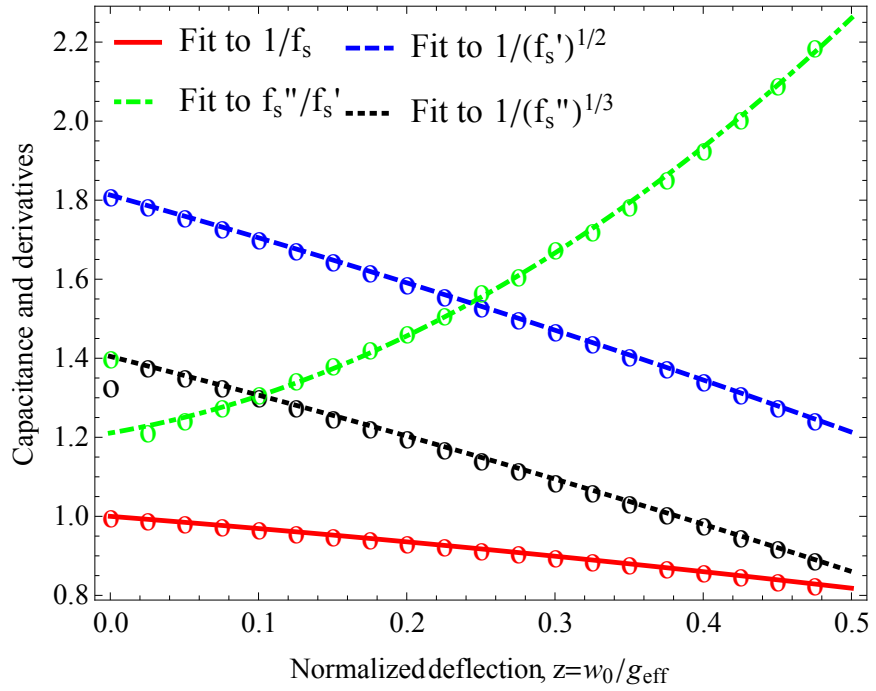

Fig. 6. Capacitance for the square plate and its derivatives. The circles show the numerically found solutions and the curves the second order fits.

the stable position, the effective spring constant, the pull-in voltage and the pull-in distance, $f_{s}^{\prime}(\eta), f_{s}^{\prime \prime}(\eta), 1 / f_{s}^{\prime \prime}(\eta)$ and $f_{s}^{\prime}(\eta) / f_{s}^{\prime \prime}(\eta)$ needs to be used. For the parallel plate, using the normalized capacitance from (38) to obtain the shape function for this plate type, the expressions will have the form

$$
\begin{aligned}
& f_{s}(\eta)=\frac{1}{1-\eta}, \quad f_{s}^{\prime}(\eta)=\frac{1}{(1-\eta)^{2}}, \quad f_{s}^{\prime \prime}(\eta)=\frac{2}{(1-\eta)^{3}}, \\
& 1 / f_{s}^{\prime \prime}(\eta)=\frac{1}{2}(1-\eta)^{3}, \quad f_{s}^{\prime}(\eta) / f_{s}^{\prime \prime}(\eta)=\frac{1}{2}(1-\eta) .
\end{aligned}
$$

The equations are then rearranged and plotted to be able to fit second order polynomials to the numerically found solution. This way, the following can be used as approximations for the square plate capacitance in the equations in the next sections

$$
\begin{aligned}
& f_{s}(\eta)=\frac{1}{1-0.296 \eta-0.136 \eta^{2}} \\
& f_{s}^{\prime}(\eta)=\frac{1}{\left(1.813-1.050 \eta-0.299 \eta^{2}\right)^{2}} \\
& f_{s}^{\prime \prime}(\eta)=\frac{1}{\left(1.405-0.953 \eta-0.271 \eta^{2}\right)^{3}} \\
& 1 / f_{s}^{\prime \prime}(\eta)=\left(1.405-0.953 \eta-0.271 \eta^{2}\right)^{3} \\
& f_{s}^{\prime}(\eta) / f_{s}^{\prime \prime}(\eta)=\frac{1}{1.211+0.647 \eta+2.906 \eta^{2}}
\end{aligned}
$$

Using these will result in deviations from the numerical solution of less than $0.05 \%, 0.08 \%, 0.3 \%$ and $2 \%$, respectively. Note that the polynomial fits are only valid for relative deflections of $0-0.5$, e.g. below pull-in. A plot showing how the polynomials are fitted can be seen in Fig. 6, where the circles show the numerically found solutions for the capacitance and its derivatives and the curves show the fits.

Fig. 7 shows the total capacitance normalized to the total capacitance with no deflection, $C_{\mathrm{t}} / C_{\mathrm{t} 0}$, versus the relative deflection, $\eta$, for all three plate types. For the square plate shape function, the numerically found solution is shown in the plot. It is seen that when normalized, the circular and square plates have similar capacitance responses. For example, 


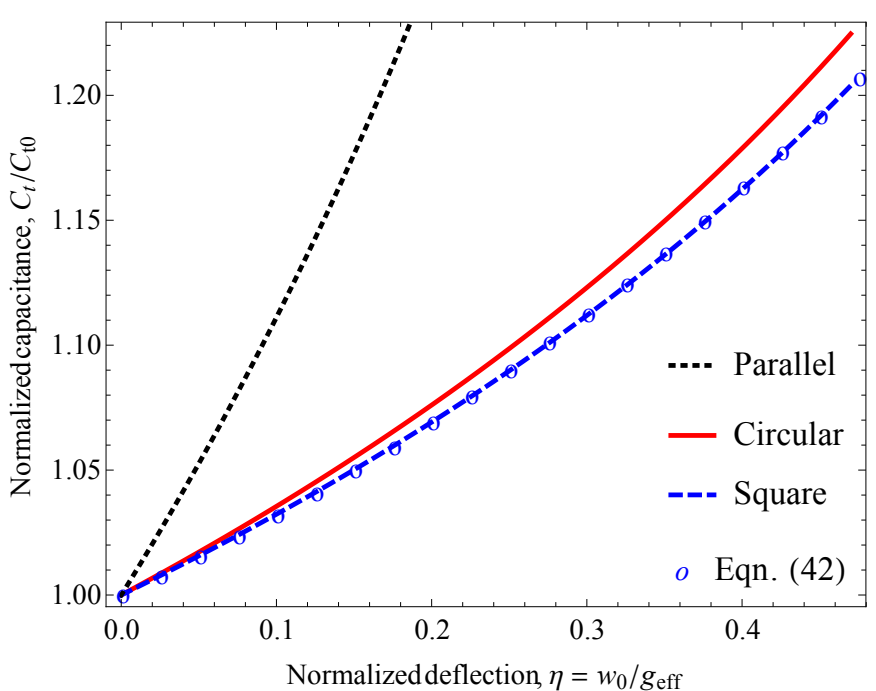

Fig. 7. Normalized total capacitance versus normalized deflection for a circular (39) and a square plate (40). The parallel plate solution (38) shown for comparison. The polynimial solution for the square plate is shown as the circles.

at a relative deflection of 0.4 the deviation is $1.4 \%$ between the square and circular capacitance, whereas using the parallel plate approximation results in a much larger difference as seen in the figure. For the square plate the polynomial solution from (42) is also shown in Fig. 7 as the circles.

\section{B. Energy calculations}

The total strain energy is calculated by integrating the strain energy density using (6) and (9). Having a thin plate, we can assume plane stress and the expression becomes

$$
U_{\mathrm{s}}=\frac{1}{2} \iiint\left(\sigma_{1} \varepsilon_{1}+\sigma_{2} \varepsilon_{2}+\sigma_{6} \varepsilon_{6}\right) \mathrm{d} x \mathrm{~d} y \mathrm{~d} z,
$$

where the strains are given by

$\epsilon_{1}=-z \frac{\partial^{2} w(x, y)}{\partial x^{2}}, \epsilon_{2}=-z \frac{\partial^{2} w(x, y)}{\partial y^{2}}, \epsilon_{6}=-2 z \frac{\partial^{2} w(x, y)}{\partial x \partial y}$.

The energy due to the externally applied pressure is calculated as minus the work performed (i.e. force times length, here pressure times area times length) when deflecting the plate

$$
U_{\mathrm{p}}=-\iint p w(x, y) \mathrm{d} x \mathrm{~d} y .
$$

The electrostatic energy is expressed through the charge $Q$ or applied voltage $V$, the vacuum permittivity $\epsilon_{0}$, gap height $g_{\text {eff }}$ and the total capacitance $C_{\mathrm{t}}$ of the device

$$
\begin{aligned}
U_{\mathrm{e}} & =-\frac{1}{2} V^{2} C_{\mathrm{t}} \\
& =-\frac{1}{2} V^{2} \iint \frac{\epsilon_{0}}{g_{\text {eff }}-w(x, y)} \mathrm{d} x \mathrm{~d} y
\end{aligned}
$$

The capacitance inserted during the second equalization in (50) is valid for all plate geometries, if the right expression for the deflection is used in each case. It can be seen how the deflection of the plate appears, and therefore, the plate geometry and the anisotropy of the plate is included through the deflection.

For a circular plate (47) becomes

$$
\begin{aligned}
U_{\mathrm{s}, \mathrm{circ}} & =\frac{1}{2} \int_{-h / 2}^{h / 2} \int_{0}^{2 \pi} \int_{0}^{a} r\left(\sigma_{1} \varepsilon_{1}+\sigma_{2} \varepsilon_{2}+\sigma_{6} \varepsilon_{6}\right) \mathrm{d} r \mathrm{~d} \theta \mathrm{d} z \\
& =\frac{h^{3} \pi w_{0}^{2}}{9 a^{2}}\left(3 C_{11}^{\mathrm{eff}}+2 C_{12}^{\mathrm{eff}}+3 C_{22}^{\mathrm{eff}}+4 C_{33}^{\mathrm{eff}}\right)
\end{aligned}
$$

and (49) and (50) become [2]

$$
\begin{aligned}
& U_{\mathrm{p}, \text { circ }}=-\int_{0}^{a} 2 \pi p r w d r=-\frac{1}{3} \pi p a^{2} w_{0} \\
& U_{\mathrm{e}, \mathrm{circ}}=-\frac{1}{2} C_{\mathrm{t}} V^{2}=-\frac{1}{2} V^{2} C_{\mathrm{t} 0} \sqrt{\frac{1}{\eta}} \operatorname{arctanh} \sqrt{\eta} .
\end{aligned}
$$

Using (11) it can be seen that the strain energy can be written in terms of the effective flexural rigidity

$$
U_{\mathrm{s}, \mathrm{circ}}=\frac{h^{3} \pi w_{0}^{2}}{9 a^{2}}\left(3+k_{2}+3 k_{4}\right) \frac{12 D_{\mathrm{a}}}{h^{3}}=\frac{32 \pi D_{\mathrm{eff}} w_{0}^{2}}{3 a^{2}}
$$

By changing the flexural rigidity, it is possible to easily switch between isotropic and anisotropic calculations in (54).

Comparing (54), (51), and (52) with (29), it can be seen that for the circular plate the general spring constant and the effective area are given by

$$
\begin{aligned}
k_{0, \text { circ }} & =\frac{2 \cdot 32 D_{\mathrm{eff}} \pi}{3 a^{2}}=\frac{64 D_{\mathrm{eff}} \pi}{3 a^{2}} \\
A_{\mathrm{eff}, \text { circ }} & =\frac{1}{3} \pi a^{2} .
\end{aligned}
$$

For the square plate, only the most common case with a highly doped plate on silicon (001) substrate aligned to the $\langle 110\rangle$ direction is considered. Using the deflection from (19), the strain energy for the square plate becomes

$$
\begin{aligned}
U_{\mathrm{s}, \mathrm{sq}} & =\frac{1}{2} \int_{-h / 2}^{h / 2} \int_{-L}^{L} \int_{-L}^{L}\left(\sigma_{1} \varepsilon_{1}+\sigma_{2} \varepsilon_{2}+\sigma_{6} \varepsilon_{6}\right) \mathrm{d} x \mathrm{~d} y \mathrm{~d} z \\
& =\frac{4096 h^{3} w_{0}^{2}}{4729725 L^{2}}\left(\gamma_{1} C_{11}^{\mathrm{eff}}+2 \gamma_{2} C_{12}^{\mathrm{eff}}+\gamma_{1} C_{22}^{\mathrm{eff}}+4 \gamma_{2} C_{33}^{\mathrm{eff}}\right), \\
\gamma_{1} & =\left(1001+468 \beta+476 \beta^{2}\right), \quad \gamma_{2}=26\left(11+2 \beta^{2}\right) .
\end{aligned}
$$

Using the value for $\beta_{\text {high }}, \gamma_{1}=1138.5$ and $\gamma_{2}=288.9$. Inserting the plate coefficients from (11) into (57), it can be seen that the strain energy can be written in terms of the plate coefficients and the anisotropic flexural rigidity

$$
U_{\mathrm{s}, \mathrm{sq}}=\frac{49152}{4729725}\left(\gamma_{1}+\gamma_{2} k_{2}+\gamma_{1} k_{4}\right) \frac{D_{\mathrm{a}} w_{0}^{2}}{L^{2}},
$$

Using the values from Table II, the strain energy for the square plate of silicon (001) aligned to the $\langle 110\rangle$ direction becomes

$$
U_{\mathrm{s}, \mathrm{sq}, \mathrm{Si}(001),[110]}=\xi_{\mathrm{s}} \frac{h^{3} w_{0}^{2}}{L^{2}},
$$

where the constant is $\xi_{\mathrm{s}}=385.637 \mathrm{GPa}$.

The energy contribution from applied pressure (49) is for this case given by

$$
\begin{aligned}
& U_{\mathrm{p}, \mathrm{sq}}=-\int_{-L}^{L} \int_{-L}^{L} p w(x, y) d x d y \\
& U_{\mathrm{p}, \mathrm{sq}, \operatorname{Si}(001),[110]}=-\xi_{\mathrm{p}} p L^{2} w_{0}
\end{aligned}
$$


where $\xi_{\mathrm{p}}=1.215$.

The electrostatic energy can in this square plate case not be found exact as an approximation is needed for the total capacitance. Using the result from (40) this energy contribution can be expressed as

$$
U_{\mathrm{e}, \mathrm{sq}}=-\frac{1}{2} V^{2} C_{\mathrm{t} 0} f_{s}(\eta) \text {. }
$$

Comparing (59) and (61) with (29), it is seen that for the square plate the general spring constant and the effective area are given by

$$
\begin{aligned}
k_{0, \mathrm{sq}} & =\frac{2 \cdot \xi_{\mathrm{s}} h^{3}}{L^{2}}=\frac{2 h^{3} \xi_{\mathrm{s}}}{L^{2}} \\
A_{\mathrm{eff}, \mathrm{sq}} & =\xi_{\mathrm{p}} L^{2} .
\end{aligned}
$$

\section{Stable position}

Using the expressions (52)-(54) for the energies and the equation for the stable position (31), the stable position for the circular plate becomes

$$
\begin{aligned}
& V_{\text {stable,circ }}= \\
& \sqrt{\frac{-256 g_{\mathrm{eff}} \eta^{3 / 2}\left(-a^{4} p \pi / 64+D_{\mathrm{eff}} \pi \eta g_{\mathrm{eff}}\right)(-1+\eta)}{3 a^{2} C_{\mathrm{t} 0}(-\operatorname{arctanh}[\sqrt{\eta}]+\eta \operatorname{arctanh}[\sqrt{\eta}]+\sqrt{\eta})}} .
\end{aligned}
$$

which is found in a similar way as in [2], [8].

A comparison of the stable position found using the anisotropic approach, (65), and measurements on a fabricated device can be found in Section VII.

For the square plate, combining the expressions in (59), (61) and (62), the stable position for the highly doped square plate on silicon (001) substrate aligned to the $\langle 110\rangle$ direction can be found by (31)

$$
V_{\text {stable,sq }}=\sqrt{\frac{2 g_{\mathrm{eff}}\left(-L^{4} p \xi_{\mathrm{p}}+2 h^{3} \eta \xi_{\mathrm{s}} g_{\mathrm{eff}}\right)}{C_{\mathrm{t} 0} L^{2} f_{s}^{\prime}(\eta)}} .
$$

For an easy approximation (43) can be inserted into (66).

Devices with square plates were also fabricated and a comparison of the stable position found using the anisotropic approaches compared to the measured center deflection can be found in Section VII.

Originally, the CMUT was modelled by use of a parallel plate approximation [4], [5]. The parallel plate case is also included here for comparison and in this case, the stable position is

$$
V_{\text {stable,parallel }}=\sqrt{\frac{2(-1+\eta)^{2} g_{\mathrm{eff}}\left(-A p+k \eta g_{\mathrm{eff}}\right)}{C_{\mathrm{t} 0}}} .
$$

From the static analysis, it is possible to present a set of general design plots for CMUTs by using adequate normalizations. Hereby, the results for circular, square and parallel plates can be compared. For specific device behavior, the equations for zero applied pressure or voltage can be used to eliminate the normalizations. These expressions are derived in section VI-E.

Fig. 8 shows the stable position of the plate for varying bias voltages. The bias voltage is normalized to the pull-in voltage at zero applied pressure $V / V_{\mathrm{PI}, \mathrm{p} 0}$ and the deflection to the

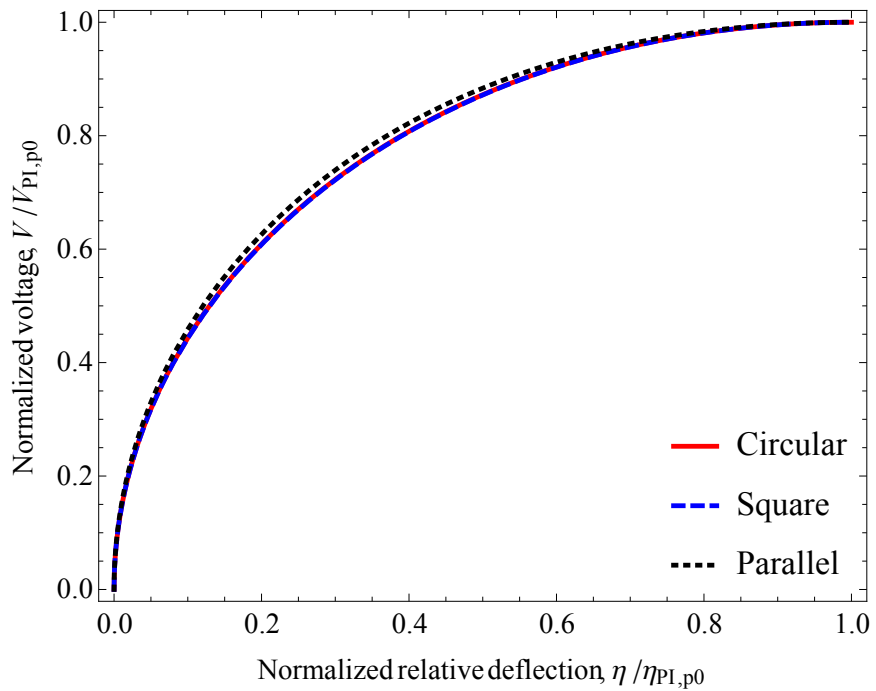

Fig. 8. Stable voltage normalized to pull-in voltage at zero applied pressure versus relative center deflection normalized to the pull-in distance at zero applied pressure for circular (65), square (66) and parallel plates (67).

pull-in distance at zero applied pressure $\eta / \eta_{\mathrm{PI}, \mathrm{p} 0}$. It is seen that the circular and square plate give almost identical results, whereas the parallel plate has a slight deviation. At $80 \%$ of pull-in, which is where the CMUT is usually designed to operate, the deviation of the square plate result compared to the circular plate result is only $0.01 \%$. For the parallel plate the deviation is $0.3 \%$ compared to the circular plate result. It should be noted that the result for the circular plate in Fig. 8 is similar to a previously shown figure in [8] which, however, does not show the parallel and square plate cases.

\section{Spring constant}

As mentioned earlier, the effective spring constant can be found by performing the double differentiation of the total potential energy with respect to center deflection, see (32). The generalized spring constant can be identified from the strain energy for both circular and square plates, (55) and (63), and for the parallel plate the spring constant is simply just $k$. All these expressions can be inserted into the generalized effective spring constant (32) to obtain the effective spring constant for each plate type. The effect of spring softening is easily seen in (32) as the second term and it is seen to depend on the capacitance. Furthermore, it is seen that the spring constant at zero applied voltage is the generalized spring constant.

In Fig. 9, the effective spring constant relative to the spring constant at zero applied voltage $k_{\text {eff }} / k_{0}$ is shown versus the normalized relative deflection $\eta / \eta_{\mathrm{PI}, \mathrm{p} 0}$ (lower axis) or normalized voltage (upper axis). The spring softening effect is clearly seen as the effective spring constant becomes smaller when the deflection and bias voltage increases. Again the circular and square plate behave almost identical and the parallel plate approximation differs from the two. Operating at $80 \%$ of pull-in the deviation between square and circular plate results is $0.47 \%$ and for the parallel plate it is $12.5 \%$.

In general, it is seen from Figs. 8 and 9 that the overall behaviour of the CMUT is well captured by both the more 


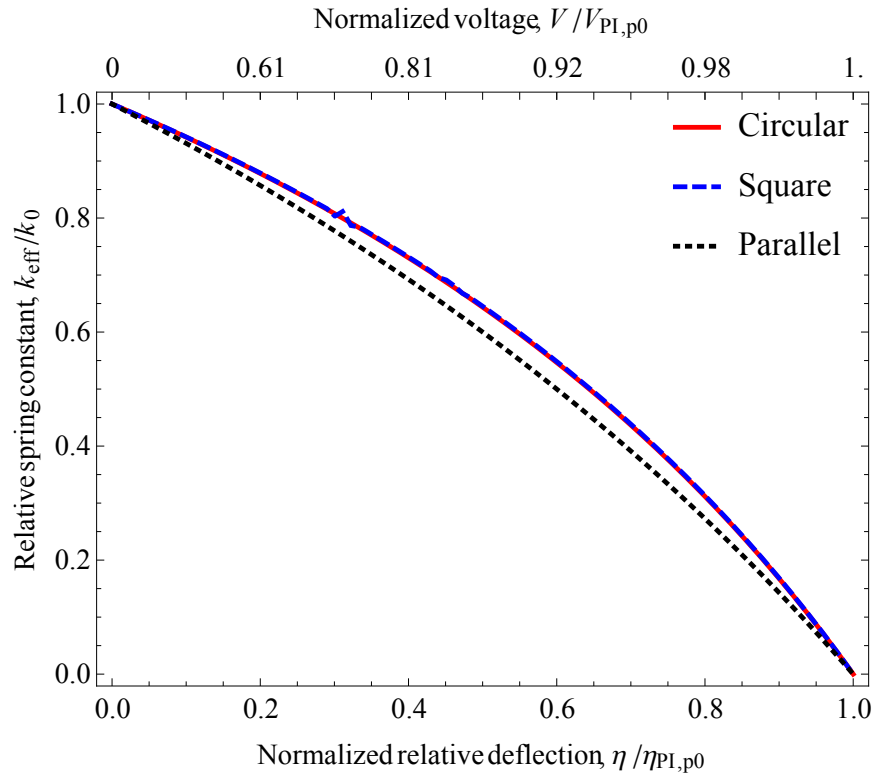

Fig. 9. Normalized effective spring constant versus relative center deflection normalized to the pull-in distance for circular, square and parallel plates.

accurate results for the circular and square plates but also by the parallel plate approximation. The difference lies in the normalizations i.e. the pull-in point calculation which is different for each case when using the actual shape of the deflection. The anisotropic effects are included through these as well. This means that practically the simple expressions can be used to model the CMUTs with good approximations, if using the specific de-normalizations for each plate type.

\section{E. Pull-in}

For the parallel plate, the pull-in distance at zero applied pressure is given by $\eta_{\mathrm{PI}, \mathrm{p} 0 \text {,parallel }}=1 / 3$. The corresponding pull-in voltage is

$$
V_{\mathrm{PI}, \mathrm{p} 0, \text { parallel }}=\sqrt{\frac{8 k g_{\mathrm{eff}}^{2}}{27 C_{\mathrm{t} 0}}} .
$$

The pressure dependence on the pull-in distance can be found analytically for this plate type and is given by

$$
\eta_{\mathrm{PI}, \text { parallel }}=1 / 3+2 / 3 p_{\mathrm{r}}
$$

where the relative pressure is given by $p_{\mathrm{r}}=p A /\left(g_{\mathrm{eff}} k\right)$. The relative pressure is the applied pressure normalized to the pressure it takes to deflect the plate the size of the effective gap, $p_{\mathrm{g}}$. Fig. 10 shows the linear dependence of the pressure on the pull-in distance, (69), as the black dotted curve. The pressure dependent pull-in voltage can for the parallel plate also be calculated analytically and is given by

$$
V_{\mathrm{PI}, \text { parallel }}=\frac{\left(-A p+k g_{\mathrm{eff}}\right)^{3}}{27 C_{\mathrm{t} 0} k^{2} g_{\mathrm{eff}}} .
$$

It is seen that the influence of the pressure on the pull-in distance, and thus also the pull-in voltage, is dependent on the geometry of the device. Defining the relative pull-in voltage as

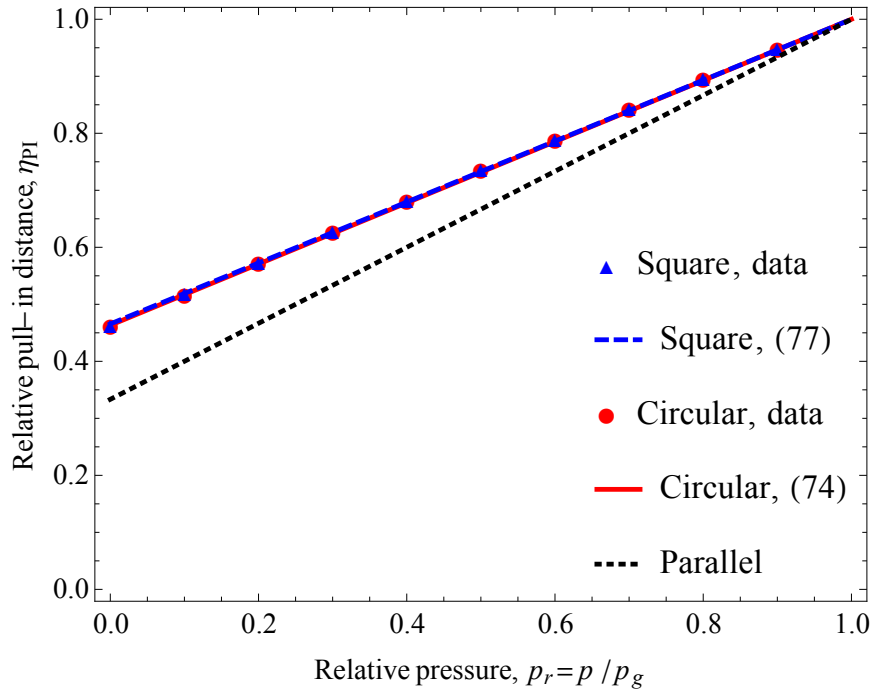

Fig. 10. Pull-in distance versus relative pressure. Circles are the full calculation for circular plates, triangles the full model for square plates, the red solid curve a fit for the circular plate (74), blue dashed curve a fit for the square plate (77) and black dotted the analytical expression for the parallel plate (69).

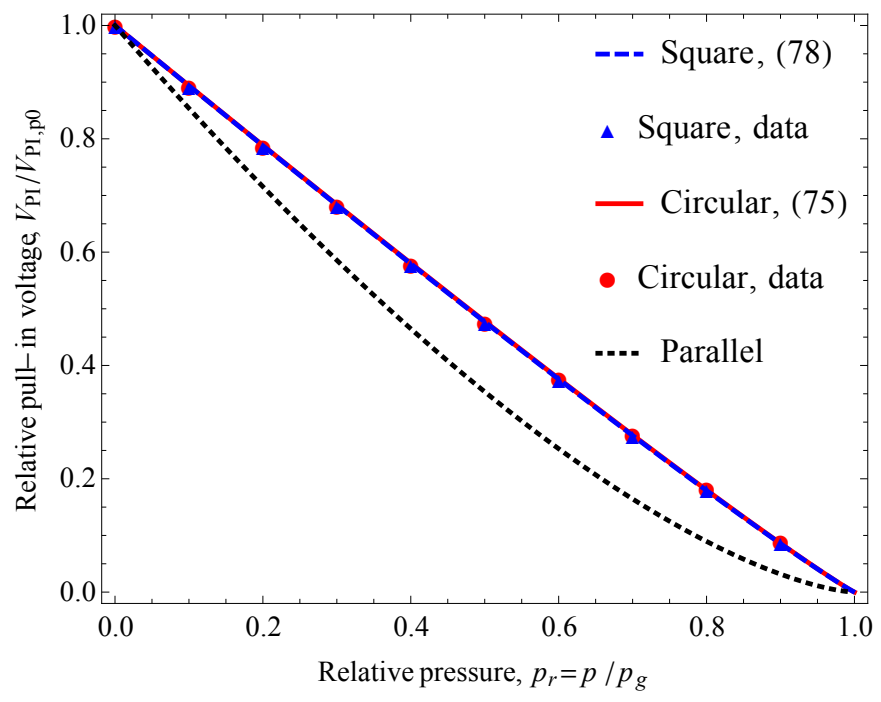

Fig. 11. Pull-in voltage relative to pull-in voltage for zero applied pressure versus relative pressure. Circles are the full calculation for circular plates, triangles the full model for square plates, the red solid curve a fit for the circular plate (75), blue dashed curve a fit for the square plate (78) and black dotted the analytical expression for the parallel plate (71).

$V_{\mathrm{rel}}=V_{\mathrm{PI}} / V_{\mathrm{PI}, \mathrm{p} 0}$ and using (70) and (68), the relative pull-in voltage for the parallel plate yields

$$
V_{\text {rel, parallel }}=\left(1-p_{\mathrm{r}}\right)^{(3 / 2)} \text {. }
$$

Fig. 11 shows a comparison of the relative voltage versus the relative pressure with a black dotted curve for the parallel plate.

Looking at the circular plate and the special case where the applied pressure is zero, the relative pull-in distance becomes $\eta_{\mathrm{PI}, \mathrm{p} 0 \text {, circ }}=0.463$ from (34). With this pull-in distance inserted into (65), the pull in voltage at zero applied pressure 
for the circular plate becomes

$$
V_{\mathrm{PI}, \mathrm{p} 0, \mathrm{circ}}=\sqrt{\frac{89.4459 D_{\mathrm{eff}} g_{\mathrm{eff}}^{2}}{a^{2} C_{\mathrm{t} 0}}} .
$$

To find the influence of the pressure on the pull-in distance, (34) is evaluated for varying values of the pressure. The result can be seen as red points in Fig. 10. As also observed by [2], [8], the influence of the pressure on the pull-in distance is found to be linear as for the parallel plate. The expression can be found by considering the boundary conditions $\eta_{\mathrm{PI}}(0)=$ $\eta_{\mathrm{PI}, \mathrm{p} 0}$ and $\eta_{\mathrm{PI}}(1)=1$. Using these conditions the expression for the pressure dependent relative pull-in distance becomes

$$
\eta_{\mathrm{PI}}=\eta_{\mathrm{PI}, \mathrm{p} 0}+\left(1-\eta_{\mathrm{PI}, \mathrm{p} 0}\right) p_{\mathrm{r}}
$$

where the relative pressure is given by $p_{\mathrm{r}}=p / p_{\mathrm{g}}=$ $p a^{4} /\left(64 g_{\text {eff }} D_{\text {eff }}\right)$ for the circular plate. Inserting $\eta_{\mathrm{PI}, \mathrm{p} 0, \text { circ }}=$ 0.463 for the circular plate yields

$$
\eta_{\mathrm{PI}, \mathrm{circ}}=0.463+0.537 p_{\mathrm{r}} .
$$

Eqn. (74) is plotted as the red solid curve in Fig. 10. The maximum deviation between the expression and the data points is $0.6 \%$. Compared to the parallel plate solution the difference in pull-in distance at zero applied pressure is clearly observed. Furthermore, note that (73) also applies for the parallel plate as seen in (69).

To see how the pressure affects the pull-in voltage for the circular plate, the relative pull-in voltage is again considered. For simplicity, the equation for the pressure dependent pull-in voltage is not shown, but it is found from the pull-in distance, (74), inserted into the stable position, (65). The resulting equation is evaluated for varying values of pressure and this is shown as red dots in Fig. 11. It is seen that the pull-in voltage decreases for increasing external pressure as expected, since the plate is deflected due to the applied pressure. To follow the analytical expression obtained for the parallel plate, a fit was made to an expression having the same form as this analytical result $V_{\text {rel }}=\left(1-p_{\mathrm{r}}\right)^{(K \cdot 3 / 2)}$, where $K$ is the fitted parameter. The result from fitting is

$$
V_{\text {rel,circ }}=\left(1-p_{\mathrm{r}}\right)^{(0.710 \cdot 3 / 2)}
$$

Using this fit a maximum deviation of only $3.9 \%$ is obtained. Also for the pull-in voltage, a difference is observed between the the parallel and circular plate.

To expand this pull-in investigation to square plates as well, the same procedure as for the circular plates is followed. For the square case, the pull-in distance in the special case of zero applied pressure becomes $\eta_{\mathrm{PI}, \mathrm{p} 0 \text {,sq }}=0.466$ which is very close to the circular plate pull-in distance. The corresponding pull-in voltage is

$$
V_{\mathrm{PI}, \mathrm{p} 0, \mathrm{sq}}=\sqrt{\frac{2.95118 g_{\mathrm{eff}}^{2} h^{3} \xi_{\mathrm{s}}}{C_{\mathrm{t} 0} L^{2}}} .
$$

To find the influence of the pressure on the pull-in distance for the square plate it was calculated for different pressures and plotted as triangular points in Fig. 10. As for the two other plate geometries, the influence of the pressure on the pull-in distance is found to be linear and using (73) it can be described as

$$
\eta_{\mathrm{PI}, \mathrm{sq}}=0.466+0.534 p_{\mathrm{r}}
$$

where the relative pressure for the square plate is given by $p_{\mathrm{r}}=0.021961 p L^{4} /\left(g_{\text {eff }} D_{\mathrm{a}}\right)$. Eqn. (77) is shown as a dashed blue line in Fig. 10. The maximum deviation between the fit and the data points for the square plate is $0.7 \%$.

In Fig. 11, it is seen how the pressure affects the pull-in voltage for the square plate shown as triangular points and a fit with a dashed blue line. The calculation method is the same as for the circular plate, and the same behavior is also observed. A fit of the data points to an expression of the same form as for the parallel plate case yields

$$
V_{\text {rel,sq }}=\left(1-p_{\mathrm{r}}\right)^{(0.712 \cdot 3 / 2)},
$$

resulting in a maximum deviation of $1.7 \%$.

\section{MEASUREMENTS}

To compare the anisotropic approach for modeling CMUTs to measurements for further validation of the theory for both circular and square plates, devices with both plate types were fabricated using a fusion bonding method [24]. The dimensions of the devices can be seen in Table V.

Measurements of the stable position (presented as the deflection in the center of the plate) for increasing bias voltage were performed on the fabricated devices. The deflections were measured as area scans with a Sensofar PLu Neox 3D Optical Profiler using white light interferometry.

Fig. 12 shows the measurements of the circular plate device. It is seen how the center deflection varies with the applied voltage and how it deflects more when approaching the pullin voltage as expected. The center deflection for the measurements is found as the average of 10 cells and gray shaded areas corresponds to plus/minus two standard deviations. For the circular device there was some uncertainty in the final gap height due to the fabrication method. Because of this it was not possible to plot the theoretical stable position for a circular plate, (65), together with the measurements. Instead, a fit was made which is shown as the theoretical curve in Fig. 12. From the fit, a gap height of $457 \mathrm{~nm}$ was found, and it is seen that the expression captures the behavior of the device very well. With this gap, the theoretical curve is within the uncertainty interval of the measurements. Also, the pull-in voltage is in good agreement with the experimentally found value, as it was measured to be $140 \mathrm{~V}$, compared to an expected value of $138 \mathrm{~V}$ from the anisotropic model (75).

Measurements with a DC voltage applied were also performed for the square plate and the results are shown in Fig. 13. The center deflection for the measurements is found as the average of 10 cells and the gray shaded areas correspond to plus/minus two standard deviations. The theoretical curve is made from the stable position analysis and is for this case plotted directly as the gap height was known from this fabrication run. It is seen that the anisotropic theory matches well with the measurement as it is within the error margin. Also, the pull-in voltages are in good agreement as it was 


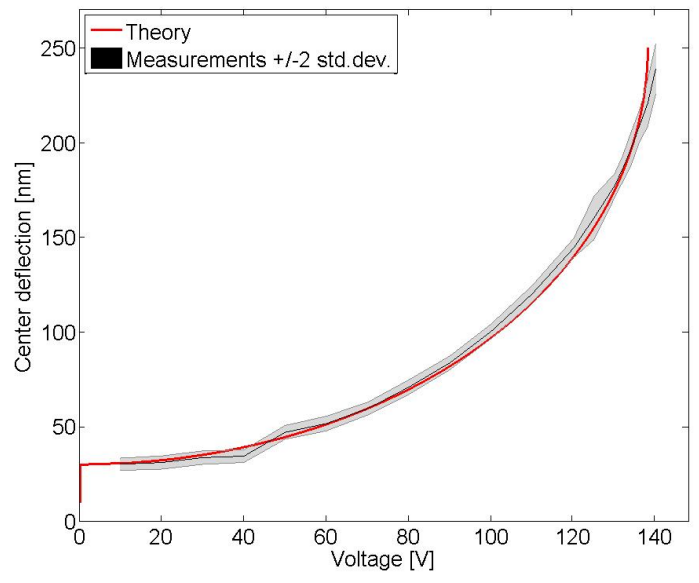

Fig. 12. Measured center deflection for increasing bias voltage together with theoretical curves for a circular plate (65).

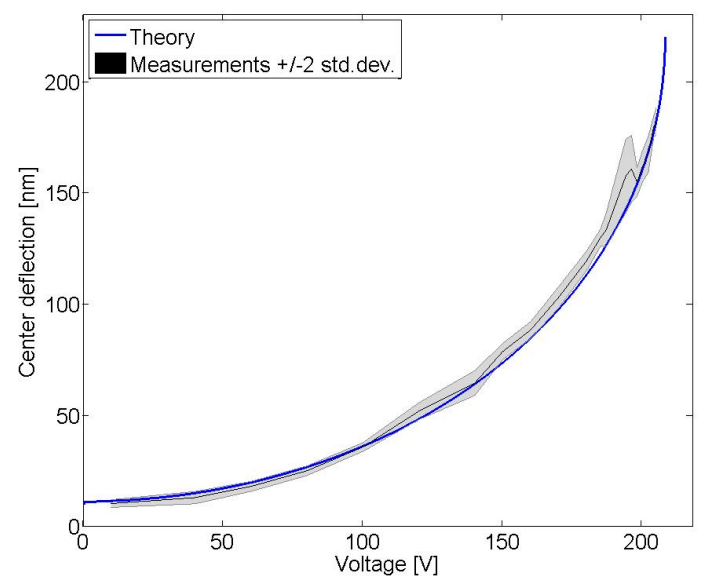

Fig. 13. Measured center deflection for increasing bias voltage together with theoretical curves for a square plate (66).

measured to be $206 \mathrm{~V}$, compared to an expected value of $201 \mathrm{~V}$ from the anisotropic model (78).

\section{CONCLUSION}

In this paper, it was demonstrated how wafer bonded CMUTs with both circular and square plates can be analytically modelled using the full anisotropic properties of single crystalline silicon. For the circular plate, an exact solution to the plate equation was obtained, and for the square plate, the full anisotropic plate equation was solved using the Galerkin method. In this case, it is seen that the deflection simplifies by utilizing the symmetry of the silicon crystal and a compact solution is obtained for square CMUT plates on a (001) silicon substrate aligned to the [110] direction. Using this approach, the analytic plate deflections show excellent correspondence with FEM calculations and measurements. Using isotropic plate theory to calculate the deflection of anisotropic silicon plates results in deviations from FEM or measurements of up to $10 \%$. Using the anisotropic theory reduces the deviation from FEM to less than $0.3 \%$ for the circular plate and $0.1 \%$ for the square plate. Fitting the anisotropic calculated deflection for the square plate to the measurement, a deviation of only $0.07 \%$ is observed for the fitted plate parameter. The theory of multilayer plates is also applied to CMUTs, however, only a small difference will be obtained in the deflection for the typical CMUT case.

A full electrostatic analysis, including the anisotropic effects was carried out for both circular, square, and parallel plate devices. The analysis is based on energy considerations and capacitance, effective spring constant, stable position, pull-in distance, and pull-in voltage are all calculated. In the pull-in analysis, the pressure dependence is also included. The circular and square plate devices are seen to behave very similar with a difference of $0.01 \%$ for stable position and $0.47 \%$ for effective spring constant at $80 \%$ of pull-in. Using the parallel plate approximation will results in deviations of $0.3 \%$ and $12.5 \%$, respectively, at $80 \%$ of pull-in. The pressure dependence is expressed through linear fits for the pull-in distance with a maximum deviation of only $0.6 \%$ for the circular plate and $0.7 \%$ for the square plate. The pressure dependent pull-in voltage was seen to follow an exponentiation expression with maximum deviations of $3.9 \%$ and $1.7 \%$ for the circular and square plate, respectively. Using the capacitance function of the circular plate for the square plate, the maximum deviation is $1.6 \%$.

Devices with both circular and square plates were fabricated, and the stable position and pull-in voltage measured. Comparing this to to the anisotropic theory, it is seen that the theory is within the uncertainty interval of the measurements in both cases.

\section{ACKNOWLEDGEMENT}

This work was financially supported by the Danish National Advanced Technology Foundation through grants 024-2008-3 and 82-2012-4.

\section{REFERENCES}

[1] P. C. Eccardt, P. Wagner, and S. Hansen, "Analytical models for micromachined transducers - an overview," in Proc. IEEE Ultrason. Symp., 2006, pp. 572-581.

[2] I. O. Wygant, M. Kupnik, and B. T. Khuri-Yakub, "Analytically calculating membrane displacement and the equivalent circuit model of a circular CMUT cell," in Proc. IEEE Ultrason. Symp., 2008, pp. 21112114.

[3] Y. Huang, A. S. Ergun, E. Hæggström, M. H. Badi, and B. T. KhuriYakub, "Fabricating capacitive micromachined ultrasonic transducers with wafer-bonding technology," J. Microelectromech. Syst, vol. 12, no. 2, pp. 128-137, 2003.

[4] I. Ladabaum, X. Jin, H. T. Soh, A. Atalar, and B. T. Khuri-Yakub, "Surface micromachined capacitive ultrasonic transducers," IEEE Trans. Ultrason., Ferroelec., Freq. Contr., vol. 45, no. 3, pp. 678-690, 1998.

[5] A. Caronti, G. Caliano, A. Iula, and M. Pappalardo, "An accurate model for capacitive micromachined ultrasonic transducers," IEEE Trans. Ultrason., Ferroelec., Freq. Contr., vol. 49, no. 2, pp. 159-168, feb 2002.

[6] A. Lohfink and P.-C. Eccardt, "Linear and nonlinear equivalent circuit modeling of CMUTs," IEEE Trans. Ultrason., Ferroelec., Freq. Contr., vol. 52, no. 12, pp. 2163-2172, 2005.

[7] A. Nikoozadeh, B. Bayram, G. Yaralioglu, and B. Khuri-Yakub, "Analytical calculation of collapse voltage of CMUT membrane," in Proc. IEEE Ultrason. Symp., vol. 1, 2004, pp. 256-259. 
[8] H. Koymen, A. Atalar, E. Aydogdu, C. Kocabas, H. K. Oguz, S. Olcum, A. Ozgurluk, and A. Unlugedik, "An improved lumped element nonlinear circuit model for a circular CMUT cell," IEEE Trans. Ultrason., Ferroelec., Freq. Contr., vol. 59, no. 8, pp. 1791-1799, 2012.

[9] B. Ahmad and R. Pratap, "Elasto-electrostatic analysis of circular microplates used in capacitive micromachined ultrasonic transducers," IEEE Sensors Journal, vol. 10, no. 11, pp. 1767-1773, 2010.

[10] S. Timoshenko and S. Woinowsky-Krieger, Theory of Plates and Shells, 2nd ed. Mcgraw-Hill College, jun 1959.

[11] R. L. Taylor and S. Govindjee, "Solution of clamped rectangular plate problems," Communications in Numerical Methods in Engineering, vol. 20, no. 10, pp. 757-765, 2004.

[12] M. Rahman, J. Hernandez, and S. Chowdhury, "An improved analytical method to design CMUTs with square diaphragms," IEEE Trans. Ultrason., Ferroelec., Freq. Contr., vol. 60, no. 4, pp. 834-845, apr 2013.

[13] G. G. Yaralioglu, B. Bayram, A. Nikoozadeh, and B. T. P. KhuriYakub, "Finite element modeling of capacitive micromachined ultrasonic transducers," Proc. SPIE Med. Imag., vol. 5750, pp. 77-86, 2005.

[14] M. F. la Cour, T. L. Christiansen, J. A. Jensen, and E. V. Thomsen, "Modelling of CMUTs with anisotropic plates", in Proc. IEEE Ultrason. Symp., 2012, pp. 588-591.

[15] M. F. la Cour, T. L. Christiansen, C. Dahl-Petersen, K. Reck, O. Hansen, J. A. Jensen, and E. V. Thomsen, "Modeling and measurements of CMUTs with square anisotropic plates," in Proc. IEEE Ultrason. Symp., 2013, pp. 2187-2190.

[16] E. Ventsel and T. Krauthammer, Thin plates and shells: theory, analysis, and applications. Dekker, 2001.

[17] K. S. Pister and S. B. Dong, "Elastic bending of layered plates," Journal of the Engineering Mechanics Division, vol. 85, no. 4, pp. 1-10, oct 1959.

[18] S. B. Dong, K. S. Pister, and R. L. Taylor, "On the theory of laminated anisotropic shells and plates," Journal of the Aerospace Sciences, vol. 29, no. 8, pp. 969-975, aug 1962.

[19] R. E. Newnham, Properties of Materials: Anisotropy, Symmetry, Structure. Oxford University Press, USA, jan 2005.

[20] E. V. Thomsen, K. Reck, G. Skands, C. Bertelsen, and O. Hansen, "Silicon as an anisotropic mechanical material: Deflection of thin crystalline plates," Sensors and Actuators, p. submitted, 2014.

[21] J. J. Hall, "Electronic effects in the elastic constants of n-type silicon," Phys. Rev., vol. 161, no. 3, pp. 756-761, sep 1967.

[22] S. Holgate, "The transverse flexure of perforated aeolotropic plates," Proceedings of the Royal Society A: Mathematical, Physical and Engineering Science, vol. 185, no. 1000, pp. 50-69, jan 1946.

[23] F. Mbakogu and M. Pavlovic, "Bending of clamped orthotropic rectangular plates: a variational symbolic solution," Computers and Structures, vol. 77, no. 2, pp. 117-128, jun 2000.

[24] T. L. Christiansen, O. Hansen, M. D. Johnsen, J. N. Lohse, J. A. Jensen, and E. V. Thomsen, "Void-free direct bonding of CMUT arrays with single crystalline plates and pull-in insulation," Proc. IEEE Ultrason. Symp., pp. 1737-1740, 2013. 